\title{
INFLUÊNCIA DO TAMANHO DE BOLHA NA FLOTAÇÃO CATIÔNICA DO QUARTZO
}

\author{
D. C. CUNHA ${ }^{1}$, A. A. POMPEO ${ }^{1}$, C. OLIVEIRA ${ }^{2}$, L. F. ROCHA ${ }^{1}$ e R.T. RODRIGUES ${ }^{*}$ \\ ${ }^{1}$ Universidade Federal do Rio Grande do Sul (UFRGS), Departamento de Engenharia de Minas \\ ${ }^{2}$ UniLaSalle, Mestrado em Avaliação em Impactos Ambientais em Mineração. \\ rafael.teixeira@ufrgs.br*
}

Artigo submetido em novembro/2015 e aceito em dezembro/2015

DOI: $10.15628 /$ holos.2015.3681

\begin{abstract}
RESUMO
Investigações científicas na área de processamento mineral por flotação têm demonstrado que tanto partículas grossas $(\mathrm{Dp}>300 \mu \mathrm{m})$ quanto partículas finas (Dp $<10 \mu \mathrm{m})$, apresentam uma baixa recuperação. Um modo efetivo de melhorar a flotação das partículas finas é diminuir o diâmetro de bolha, promovendo um aumento no holdup e no fluxo de área superficial de bolhas (bubble surface area flux), aumentando assim a probabilidade de colisão bolha-partícula. Entretanto, um decréscimo excessivo do diâmetro de bolha pode causar
\end{abstract}

uma diminuição da capacidade de carregamento e, consequentemente, uma redução na eficiência de flotação. Assim, o objetivo deste trabalho foi avaliar a flotação de partículas de quartzo com diferentes tamanhos de bolha $(0,50 \mathrm{~mm}<\mathrm{Db}$ médio $<2,9 \mathrm{~mm})$, utilizando uma coluna de flotação minipiloto automatizada. Os resultados obtidos mostraram que a recuperação de partículas grossas $\left(D_{32}=345 \mu \mathrm{m}\right)$ e de partículas finas $\left(D_{32}=25 \mu \mathrm{m}\right)$, foram incrementadas com a diminuição do tamanho de bolha.

PALAVRAS-CHAVE: tamanho de partícula, tamanho de bolha, flotação.

\section{THE INFLUENCE OF THE BUBBLE SIZE ON THE CATIONIC FLOTATION OF QUARTZ}

\begin{abstract}
Scientific investigations in the mineral processing area by flotation have demonstrated that both coarse particles $(\mathrm{Dp}>300 \mu \mathrm{m})$ and fine particles $(\mathrm{Dp}<10 \mu \mathrm{m})$ show a small flotation efficiency. An effective way to improve the flotation of fine particles is decrease the bubble size, promoting an increase of both holdup and bubble surface area flux, thus, increasing the probability of bubbleparticle collision. However, an excessive decrease of the bubble size may cause an overloading of the bubbles and,
\end{abstract}

therefore, a decrease in flotation efficiency. Thus, the aim of this work was to evaluate the flotation of quartz particles with different bubble sizes $(0.50 \mathrm{~mm}<\mathrm{Db}$ mean $<$ $2.9 \mathrm{~mm}$ ), using an automatized mini pilot flotation column. The results obtained showed that the flotation efficiencies of coarse particles $\left(D_{32}=345 \mu \mathrm{m}\right)$ and fine particles $\left(D_{32}=25 \mu \mathrm{m}\right)$ were improved with a decrease in bubble size. 


\section{INTRODUÇÃO}

Trabalhos científicos têm demonstrado que tanto as partículas grossas quanto as partículas muito finas apresentam uma baixa eficiência de flotação devido, principalmente, a problemas de adesão e colisão, respectivamente. Um modo efetivo de melhorar a flotação das partículas finas é reduzir o tamanho das bolhas, o que corresponde a um aumento na área superficial de bolhas e portanto num aumento da probabilidade de colisão (Reay and Ratcliff, 1973; Ahmed and Jameson, 1985; Diaz-Penafiel and Dobby, 1994; Luttrell et al. 1988). O trabalho de Reay e Ratcliff (1973), usando bolhas de aproximadamente $100 \mu \mathrm{m}$ de diâmetro, forneceu a primeira evidência experimental da capacidade de melhora da flotação de partículas finas pela diminuição do tamanho de bolha. A geração de bolhas pequenas $(<100 \mu \mathrm{m})$ é particularmente importante para flotação de partículas finas $(5-30 \mu \mathrm{m}$ ) (Ahmed e Jameson, 1985; Rodrigues, 2004; Englert et al., 2011). Luttrell et al. (1988) em seus estudos de flotação de finos de carvão atribuíram uma diminuição na recuperação (de $95 \%$ para menos $40 \%$ ) ao aumento do diâmetro médio de bolha de aproximadamente $0,35 \mathrm{~mm}$ para mais de $1,2 \mathrm{~mm}$.

Embora a coleta (colisão+adesão) seja melhorada com o aumento da área superficial de bolhas provocado pela diminuição do tamanho das mesmas, este benefício pode não resultar em melhora no desempenho metalúrgico da flotação. Bolhas menores possuem menor capacidade de carregamento e menor velocidade de ascensão, assim, podem ser arrastadas se a velocidade de ascensão for menor do que a velocidade superficial do líquido no interior da coluna. Deste modo, a diminuição excessiva do tamanho de bolha pode levar a diminuição da flotação das partículas mais grossas e, portanto, uma mistura de bolhas de tamanhos variados (produzidas por métodos diferentes) pode ser necessária para produzir melhores condições de flotação (Filippov et al., 2000). Cilliers e Bradshaw (1996) concluíram que o sistema de flotação ideal (flotação seletiva de minérios) para alcançar altos teores e recuperação deve possuir bolhas pequenas na zona de polpa (zona de coleta) e uma espuma não muito estável (que coalesça) constituída de bolhas grandes para promover a drenagem. Embora o tamanho de bolha seja de grande importância na flotação, deve ficar claro que este efeito também depende de outras variáveis, especialmente do grau de agitação ou turbulência, a densidade das partículas ou agregados e do diâmetro de partículas ou agregados.

O objetivo deste trabalho foi avaliar a flotação catiônica do quartzo para diferentes tamanhos de partículas $\left(D_{32}=25\right.$ a 345,5 $\left.\mu \mathrm{m}\right)$ e diferentes tamanhos de bolha $\left(D_{32}=0,5\right.$ a $\left.2,9 \mu \mathrm{m}\right)$ utilizando uma coluna de flotação minipiloto automatizada.

\section{METODOLOGIA}

\subsection{Materiais}

A amostra de quartzo (areia quartzosa beneficiada) fornecida pela empresa Micromil Micronização e Moagem Ltda (SC/Brasil) passou pelas seguintes etapas de beneficiamento: lavagem, classificação, secagem, separação magnética e moagem a seco em moinho de seixos (pebble mill). A amostra foi separada em diferentes frações granulométricas através do peneiramento a úmido, sendo a seguir seca em estufa a $60^{\circ} \mathrm{C}$, homogeneizada e acondicionada. $\mathrm{A}$ 
Tabela 1 apresenta as faixas granulométricas separadas por peneiramento e os diâmetros característicos das frações obtidos por um granulômetro a laser (CILAS ${ }^{\circledR}$ 1064). A Figura 1 apresenta as curvas de distribuição granulométrica (difração laser) das amostras.

Tabela 1. Faixas granulométricas das amostras de quartzo utilizadas nos estudos de flotação.

\begin{tabular}{|c|c|c|c|c|c|}
\hline \multicolumn{2}{|c|}{ Faixa Granulométrica } & \multirow{2}{*}{$\mathrm{D} 10(\mu \mathrm{m})$} & \multirow{2}{*}{ D50 $(\mu \mathrm{m})$} & \multirow{2}{*}{$\mathrm{D} 90(\mu \mathrm{m})$} & \multirow{2}{*}{$\mathrm{D}_{32}(\mu \mathrm{m})$} \\
\hline Mesh Tyler & $\mathrm{mm}$ & & & & \\
\hline$-35+48 \#$ & $-420+297$ & 228,9 & 349,5 & 466,1 & 345,5 \\
\hline$-48+65 \#$ & $-297+210$ & 174,2 & 246,1 & 354,1 & 254,5 \\
\hline$-65+100 \#$ & $-210+149$ & 137,6 & 192,7 & 274,1 & 199,0 \\
\hline$-100 \#$ & -149 & 1,6 & 18,1 & 60,4 & 25,0 \\
\hline -100\# (deslamada) & -149 & 14,5 & 36,5 & 77,8 & 41,6 \\
\hline
\end{tabular}

Soluções aquosas de amina (Flotigam EDA, Clariant ${ }^{\circledR}$ ) e de Dowfroth 250 (Éter Metílico de Polipropilenoglicol) foram preparadas com água destilada para uso nos ensaios de flotação. Todos os ensaios foram realizados a temperatura ambiente $\left(\sim 25^{\circ} \mathrm{C}\right)$, com água da rede municipal de abastecimento de Porto Alegre/RS (DMAE) e pH natural $(\sim 7,5)$. Todos os ensaios foram realizados em duplicata ou triplicata.

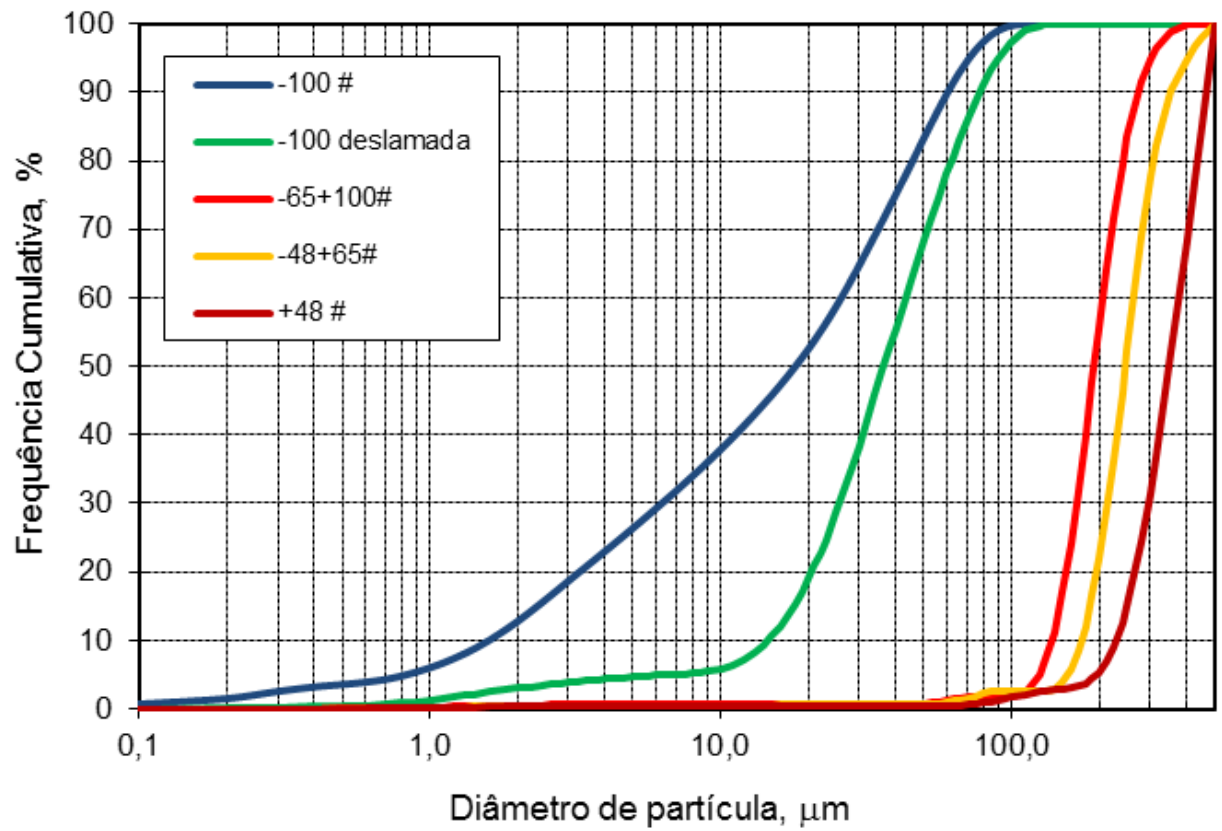

Figura 1. Distribuições granulométricas das amostras de quartzo utilizadas nos ensaios de flotação.

\subsection{Métodos}

A Figura 2 apresenta um esquema da montagem experimental. A coluna de flotação minipiloto possuía $2,75 \mathrm{~cm}$ de diâmetro interno e $210 \mathrm{~cm}$ de altura . A polpa de quartzo com $10 \%$ de sólidos em peso foi mantida sob agitação constante. A vazão de alimentação da coluna foi de 70 $\mathrm{mL} / \mathrm{min}$ e a dosagem de coletor (amina) foi realizada em linha. A coluna foi preenchida com água e alimentação iniciou após 10 minutos de operação apenas com injeção de tensoativo (DF250), tempo necessário para a estabilização do tamanho de bolha e do holdup. Cada ensaio teve duração de $1 \mathrm{~h}$ e todo o material flotado e afundado (não-flotado) foram coletados para obtenção dos resultados 
de recuperação mássica (percentagem em peso obtida no flotado). O holdup foi determinado através de medidas de pressão realizadas por dois sensores de pressão instalados na zona de coleta da coluna de flotação. Para geração de bolhas foram utilizados um tubo poroso ou um micro-venturi instalados na câmara de geração de bolhas. O tubo poroso, fabricado com pó de aço inox sinterizado, possuía medidas externas de $15 \times 30 \mathrm{~mm}$ (diâmetro x comprimento) e espessura de parede de 2,3 mm. A vazão total (água + solução de tensoativo) injetada na câmara de geração de bolhas, quando utilizado o tubo poroso, foi de $300 \mathrm{~mL} / \mathrm{min}$. O micro-venturi possuía diâmetro de orifício igual a $0,8 \mathrm{~mm}$ e foi confeccionado em poliuretano. A vazão total (água + solução de tensoativo) injetada no micro-venturi foi de $200 \mathrm{~mL} / \mathrm{min}$ e a pressão resultante foi de $147 \mathrm{kPa}$. O sistema experimental utilizado para determinação do tamanho de bolha foi o mesmo descrito no trabalho de Oliveira et al. (2011) e Pompeo et al. (2013), entretanto, foram realizadas modificações quanto a sua aplicação para permitir a medição e controle online do tamanho de bolha geradas na base de colunas de flotação.

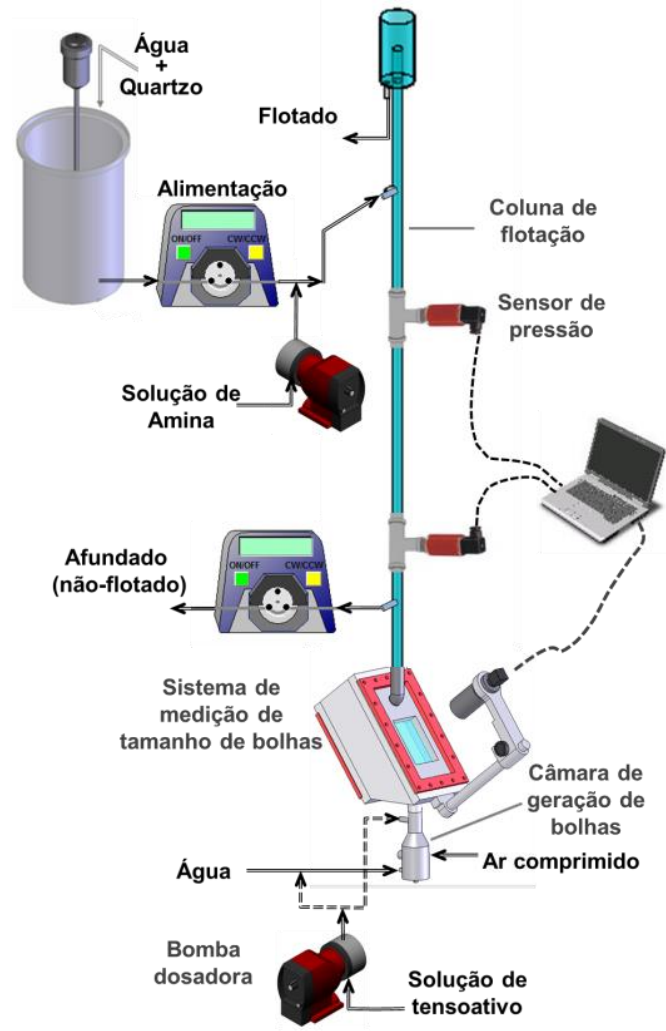

Figura 2. Montagem experimental desenvolvida para os estudos de flotação em coluna com controle online do tamanho de bolha.

Na análise das imagens foi utilizado o diâmetro de Feret médio (medido em 8 ângulos) como sendo o diâmetro de bolha. $O$ diâmetro médio adotado (calculado com base nos diâmetros de bolha obtidos da análise de imagens) foi o diâmetro médio de Sauter $\left(D_{32}\right)$, definido como o diâmetro médio volume-superfície, conforme a Equação 1.

$$
\mathrm{d}_{\text {sauter }}=\frac{\Sigma \mathrm{n}_{\mathrm{i}} \mathrm{d}_{\text {bi }}^{3}}{\Sigma \mathrm{n}_{\mathrm{i}} \mathrm{d}_{\mathrm{bi}}^{2}}
$$

onde, os parâmetros $d_{b}$ e $n$ são o diâmetro de bolha e o número de bolhas para cada classe de tamanho " $i$ ", respectivamente. 


\section{RESULTADOS E DISCUSSÕES}

Foram utilizadas três configurações para produção dos diferentes tamanhos de bolha, conforme mostra a Tabela 2. Este sistema permitiu controlar o tamanho de bolha de forma independente das demais condições operacionais.

Tabela 2. Configurações utilizadas para a produção dos diferentes tamanhos de bolha.

\begin{tabular}{ccccc}
\hline $\begin{array}{c}\text { Configuração da } \\
\text { câmara de } \\
\text { geração de } \\
\text { bolhas }\end{array}$ & Tipo de sparger & $\begin{array}{c}\text { Ponto de injeção do } \\
\text { espumante }\end{array}$ & $\begin{array}{c}\text { Concentração de } \\
\text { DF250 na câmara de } \\
\text { geração de bolhas, } \\
\text { mg/L }\end{array}$ & $\begin{array}{c}\text { Diâmetro médio de } \\
\text { bolha ( }\end{array}$ \\
\hline N.1 & Tubo poroso & mm
\end{tabular}

A Figura 3 e a Tabela 2 apresentam os tamanhos médios de bolha obtidos nos ensaios de flotação em função das dosagens de espumante (DF250) para as diferentes configurações de geração de bolha. Foi possível obter distintos tamanhos de bolha, numa faixa entre 0,5 e 2,9 mm.

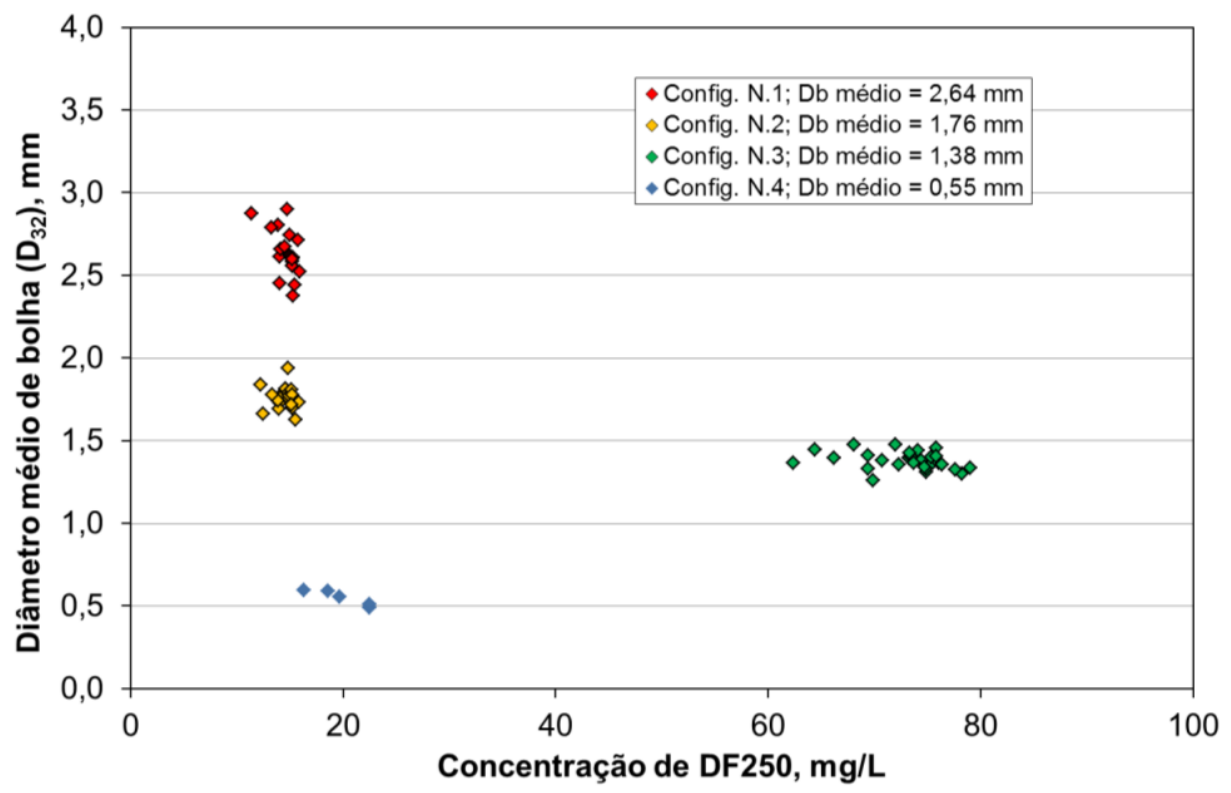

Figura 3. Efeito da concentração de espumante (DF 250) no diâmetro médio de bolha para diferentes configurações da câmara de geração de bolhas (ver Tabela 2).

A Figura 4 evidencia que para dosagens de amina superiores a $130 \mathrm{~g} / \mathrm{t}$ não se obtém incrementos significativos na recuperação. Estes ensaios foram realizados com a amostra -100\# e adotou-se esta concentração para os estudos de flotação das demais frações granulométricas. 


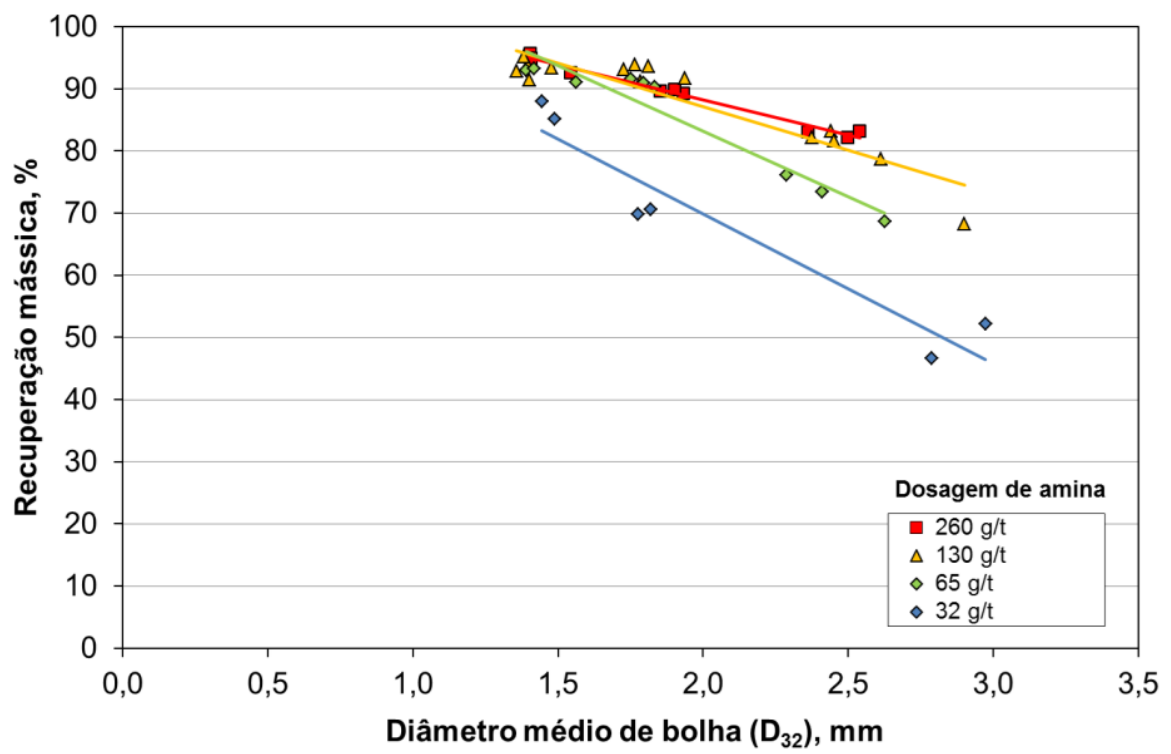

Figura 4. Efeito do tamanho de bolha na recuperação de partículas de quartzo para diferentes dosagens de Amina. Condições experimentais: Granulometria da amostra = -100 \#; pH = 7-8; Vazão de ar = $180 \mathrm{~mL} / \mathrm{min} ; \mathrm{Jg}=0,52 \mathrm{~cm} / \mathrm{s}$; Vazão de alimentação: $70 \mathrm{~mL} / \mathrm{min}$.

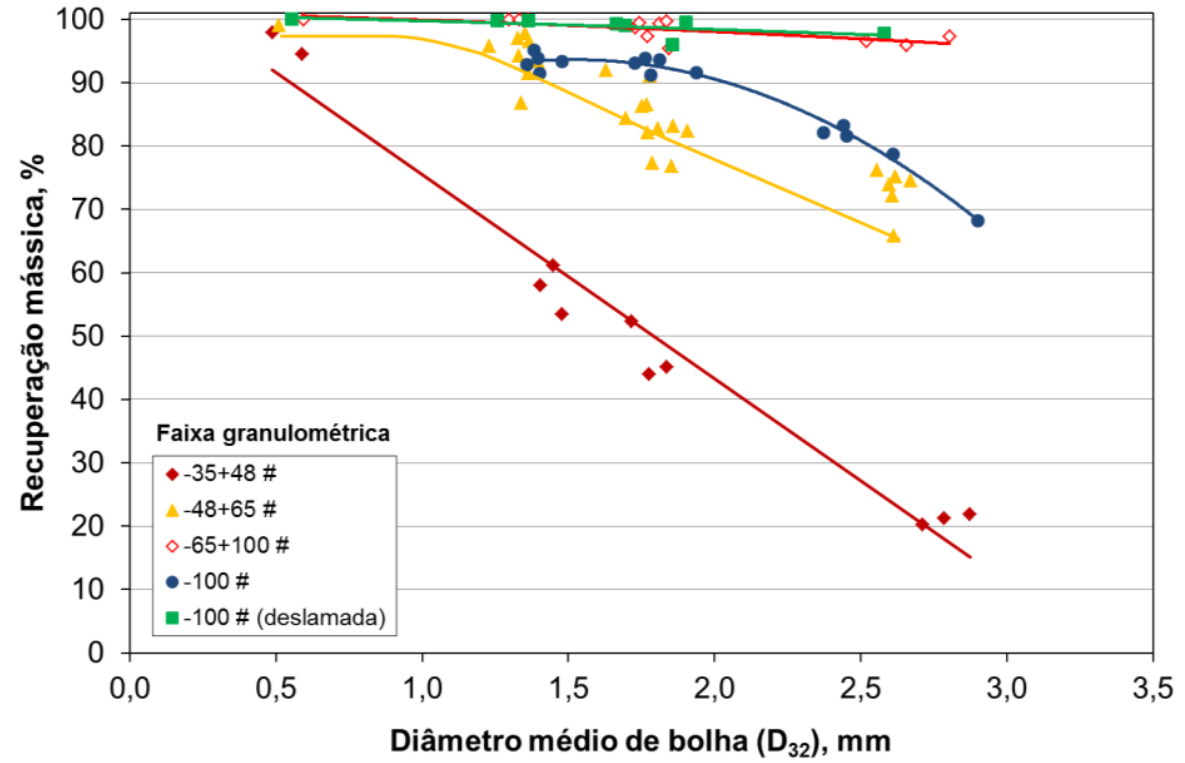

Figura 5. Efeito do tamanho de bolha na recuperação de partículas de quartzo. Condições experimentais: $\mathbf{p H}=7-8$, Vazão de ar $=180 \mathrm{~mL} / \mathrm{min}, \mathrm{Jg}=0,52 \mathrm{~cm} / \mathrm{s}$, Vazão de alimentação: $70 \mathrm{~mL} / \mathrm{min},[$ Amina] $=130 \mathrm{~g} / \mathrm{t}$. 


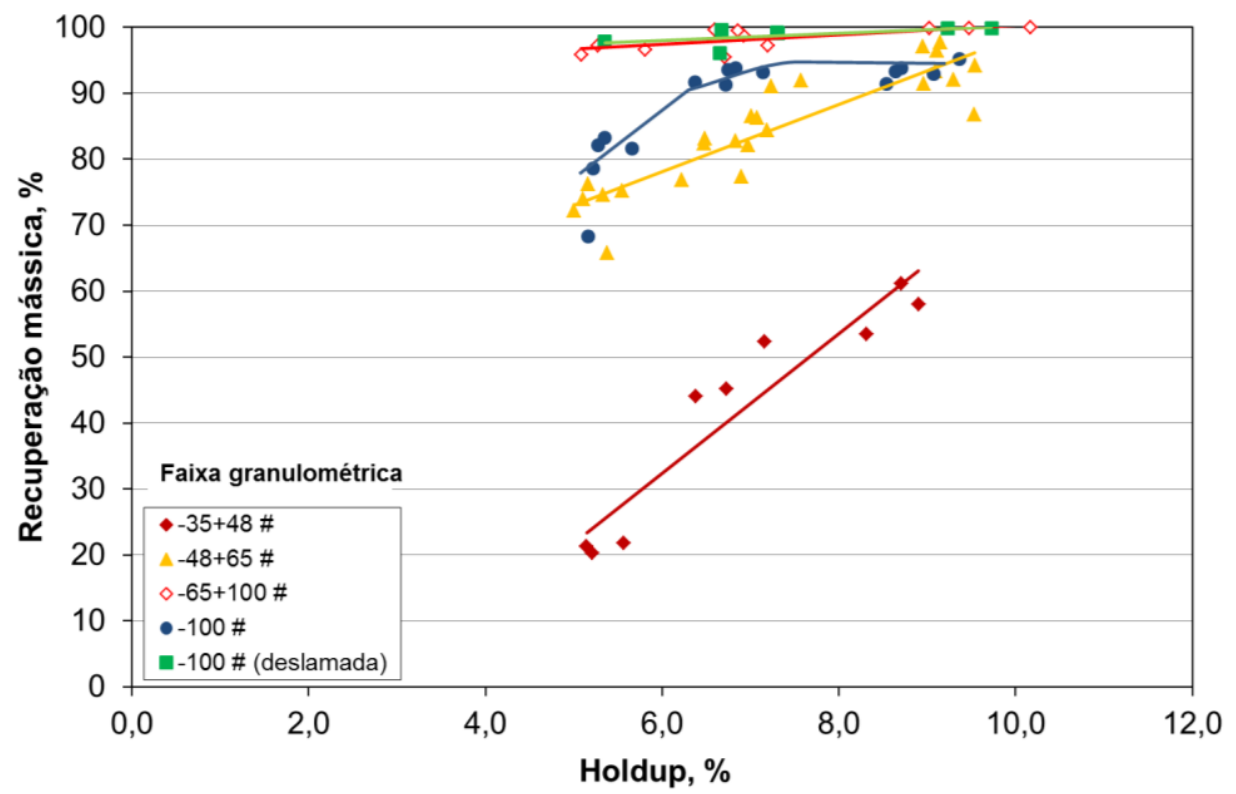

Figura 6. Efeito do holdup na recuperação de partículas de quartzo. Condições experimentais: $\mathrm{pH}=7-8, \mathrm{Vazão}$ de $\operatorname{ar}=180 \mathrm{~mL} / \mathrm{min}, \mathrm{Jg}=0,52 \mathrm{~cm} / \mathrm{s}$, Vazão de alimentação: $70 \mathrm{~mL} / \mathrm{min}$, [Amina] $=130 \mathrm{~g} / \mathrm{t}$.

Os resultados obtidos evidenciaram que a flotação, tanto das frações mais finas, quanto das frações mais grossas, foi incrementada com a diminuição do tamanho de bolhas (Fig. 5) e de forma similar com o aumento do holdup (Fig. 6). O efeito do tamanho de bolha no incremento da recuperação foi relevante para as frações $-35+48 \#,-48+65 \#$ e $-100 \#$, com ganhos da ordem de 30 a 70 \%. A recuperação das frações $-65+100 \#$ e $-100 \#$ deslamada tiveram elevada eficiência (> 95 \%) para todos os tamanhos de bolha.

Os resultados obtidos com a amostra de granulometria -100\# $\left(D_{50}=18,08 \mu \mathrm{m}\right.$ e $D_{10}=1,6$ $\mu \mathrm{m}$ ) obtiveram recuperações inferiores em relação à fração -100\# deslamada ( $D_{50}=36,45 \mu \mathrm{m}$ e $D_{10}$ $=14,5 \mu \mathrm{m}$ ), sugerindo a maior dificuldade de flotação das partículas finas e ultrafinas. Barbosa e Baltar (2012) também evidenciaram a maior dificuldade de flotação de partículas finas de quartzo utilizando célula mecânica (DENVER). Segundo os autores, a amostra com tamanho médio de $42 \mu \mathrm{m}$ flotou praticamente toda com apenas dois minutos de flotação, enquanto a amostra mais fina $\left(D_{50}=17 \mu \mathrm{m}\right)$ teve uma recuperação de apenas $25 \%$ no mesmo período. A dificuldade foi atribuída a uma menor densidade de adsorção do coletor nas partículas mais finas devido à maior área superficial. No entanto, a recuperação aumentou para aproximadamente $77 \%$ com o prolongamento do tempo de flotação e chegou a $95 \%$ com o aumento da concentração do coletor.

\section{CONCLUSÕES}

Com o sistema proposto foi possível avaliar o efeito do tamanho de bolha na flotação de partículas de quartzo. Com as configurações empregadas foi possível obter distintos tamanhos de bolha, numa faixa entre 0,5 e 2,9 $\mathrm{mm}$. Os resultados obtidos mostraram que a recuperação, tanto das frações mais finas, quanto das frações mais grossas, é incrementada com a diminuição do tamanho de bolha e, de forma similar, com o aumento do holdup. $\mathrm{O}$ efeito do tamanho de bolha no incremento da recuperação foi relevante para as frações $-35+48 \#,-48+65 \#$ e $-100 \#$, com ganhos da 
ordem de 30 a $70 \%$. A recuperação das frações $-65+100 \#$ e $-100 \#$ deslamada tiveram elevada eficiência (> 95 \%) para todos os tamanhos de bolha. A flotação da amostra -100\# obteve recuperação inferior em relação à fração -100 \# deslamada, evidenciando a menor eficiência de flotação das partículas finas e ultrafinas.

\section{REFERÊNCIAS BIBLIOGRÁFICAS}

1. AHMED, N., JAMESON, G.J. The effect of bubble size on the rate of flotation of fine particles. International Journal of Mineral Processing, v.14, n.3, p.195-215, 1985.

2. BARBOSA, J.L.O., BALTAR, C.A.M. Influência do tamanho de partícula na flotação com amina. HOLOS, v.5, p.93-100, 2012.

3. CILLIERS, J.J., BRADSHAW, D.J. The flotation of fine pyrite using colloidal gas aphrons. Minerals Engineering, v.9, n.2, p.235-241, 1996.

4. DIAZ-PENAFIEL, P., DOBBY, G.S., Kinetic studies in flotation column: bubble size effect. Minerals Engineering, v.7, n.4, p.465-478, 1994.

5. ENGLERT, A.H., RODRIGUES, R.T., RUBIO, J. Flotação de partículas ultrafinas: estado da arte e contribuição técnica sobre o ponto de mínimo na eficiência de captura bolha-partícula. In: Encontro Nacional de Tratamento de Minérios e Metalurgia Extrativa. Anais do XXIV Encontro Nacional de Tratamento de Minérios e Metalurgia Extrativa; 2011; Salvador, Brasil. p. 66-73.

6. FILIPPOV, L.O., JOUSSEMENT, R., HOUOT, R. Bubble Spargers in Column Flotation: Adaptation to Precipitate Flotation. Minerals Engineering, v.13, n.1, p.37-51, 2000.

7. LUTTRELL, G.H., WEBER, A.T., ADEL, G.T., YOON, R.H. Microbubble flotation of fine coal. In: SME Annual Meeting. Proceedings of an International Symposium of Column flotation - Column Flotation 88', Editor: Department of Materials Science and Mineral Engineering - University of California at Berkeley, 1988; January 25-28: Phoenix, Arizona. p. 205-211.

8. OLIVEIRA, C., SEGER, M.A., RODRIGUES, R.T. Desenvolvimento de um equipamento para avaliação da dispersão de bolhas em células de flotação. In: Encontro Nacional de Tratamento de Minérios e Metalurgia Extrativa. Anais do XXIV Encontro Nacional de Tratamento de Minérios e Metalurgia Extrativa; 2011; Salvador, Brasil. p. 115-124.

9. POMPEO, A.A., OLIVEIRA, C., SEGER, M.A., LOTTERMANN, M.R., RODRIGUES, R.T. Avaliação do tamanho de bolha produzido por um sparger cominco. In: Encontro Nacional de Tratamento de Minérios e Metalurgia Extrativa. Anais do XXV Encontro Nacional de Tratamento de Minérios e Metalurgia Extrativa; 2013; out. 20-24: Goiânia, Brasil. p. 61-69.

10. REAY, D., RATCLIFF, G.A. Removal of fine particles from water by dispersed air flotation: effect of bubble size and particle size on collection efficiency. Canadian Journal of Chemical Engineering, v.51, p.178-185, 1973.

11. RODRIGUES, R.T., RUBIO, J. New basis for measuring the size distribution of bubbles. Minerals Engineering, v.16, p.757-765, 2003.

12. RODRIGUES, R.T. Desenvolvimento da Técnica LTM-BSizer para a Caracterização de Bolhas e Avaliação de Parâmetros no Processo de Flotação. [Tese de Doutorado]. Programa de PósGraduação em Engenharia de Minas, Metalúrgica e de Materiais - PPGEM/UFRGS; 2004. 